Regions of interest (ROIs) were the spine, the hip, the forearm and juxtaarticular subregions of the fingers.

Results Patients with Rheumatoid arthritis showed decreased bone densities of the spine, the hip, the forearm and the juxtaarticular joint region of the fingers. This was the case in late and early stages (Larsen $0-1$ ) of the disease. The relative loss of juxtaarticular bone density was in these cases comparable to the relative loss of bone density of the spine and the hip.

Conclusion DXA revealed loss of juxtaarticular bone density in patients suffering from Rheumatoid arthritis. This could be a method for objective estimation of juxtaarticular osteoporosis in the early course of Rheumatoid arthritis.

Infection related rheumatic diseases

\section{AB0203 A CASE WITH SPINAL BRUCELLOSIS AND LUMBAR DISC HERNIATION}

R Celiker, S Bal. Department of Physical Medicine and Rehabilitation, Hacettepe University, School of Medicine, Ankara, Turkey

\subsection{6/annrheumdis-2001.714}

Background Human Brucellosis is a zoonosis and endemic in Mediterranean area. Localised osteoarticular manifestations of brucellosis include spondylitis, sacroiliitis, mono/oligoarthritis and bursitis. Coexistence of lumbar intervertebral disc herniation in spondylitis cases which do not have fever and constitutional symptoms, however, makes the diagnosis challenging.

Objectives We describe a patient with brucella spondylitis accompanying L4-5 and L5-S1 herniated nucleus pulposus (HNP).

Methods A 50 year old woman admitted to our outpatient clinic with low back pain, radiating to the lateral aspect of the left thigh lasting for 3 weeks. She did not have any history of fever, chills and constitutional symptoms. On physical examination, she was afebrile and only had tenderness over the lumbosacral region. The neurological examination revealed hypoesthesia in the right lower extremity overlapping the L5 dermatome. Straight leg raising test was positive on the right side.

Results Laboratory values were as follows: erythrocyte sedimentation rate $16 \mathrm{~mm} / \mathrm{hr}$, leucocyte count $4600 / \mathrm{mm} 3$, haemoglobin $10.4 \mathrm{gr} / \mathrm{dl}$, C-reactive protein $0.143 \mathrm{mg} / \mathrm{dl}$. Hepatic and renal function tests were normal. Seroagglutination test for brucella was $1 / 640$. Plain radiography of the lumbar region revealed L5 spondylolisthesis and narrowed disc space between L5-S1 vertebrae. Magnetic resonance imaging (MRI) disclaimed L5 spondylolisthesis, spondylolysis, L5-S1 discitis, L5-S1 HNP, bilateral foraminal stenosis and L4-L5 minimal posterior protrusion. Total body bone scan with Tc-99m sulfur colloid revealed increased uptake at the superior region of the sacrum. Oral treatment with doxycycline and rifampin was started. There was overt clinical improvement after combined antibiotic regimen for 6 weeks. After a symptom-free period of 7 weeks a relapse occurred. An oral regimen of rifampin and ofloxacin was started again. Following up with the antibiotic therapy and immobilisation with rigid lumbosacral brace, she had a dramatic reduction in low back pain and she had regressive findings of the lesion in the control MRI.

Conclusion In some areas where brucellosis is rarely seen, due to non-spesific clinical picture and low index of suspicion, disease can easily be misdiagnosed. Spinal brucellosis can be confused with disc herniation, leading to inappropriate treatment and increased morbidity. In endemic regions, coexistence with lumbar disc herniation, like in our patient, should always be in mind of the physician and clinically, diagnosing of herniation alone should not seem satisfactory all the time.

\section{AB0204 EARLY DIAGNOSIS AND FOLLOW-UP OF PYOMYOSITIS BY COMPUTED TOMOGRAPHY}

${ }^{1} \mathrm{~N}$ Kyriazis, ${ }^{2} \mathrm{G}$ Pardalis, ${ }^{2} \mathrm{E}$ Kyprizlis, ${ }^{2} \mathrm{~A}$ Papageorgiou, ${ }^{2} \mathrm{~L}$ Brianas. ${ }^{1}$ Rheumatology Department; ${ }^{2}$ First Department of Internal Medicine, General Prefectural Hospital of Karditsa, Karditsa, Greece

\subsection{6/annrheumdis-2001.715}

Background Pyomyositis is a bacterial infection of skeletal muscles, most commonly caused by staphylococcus aureus. If diagnosed early, pyomyositis can be treated with antibiotics alone. However, the diagnosis is often overlooked, or delayed because of lack of familiarity with this disease. Clinical suspicion and appropriate imaging techniques are the keys to early diagnosis.

Objectives To report a new case of pyomyositis, demonstrating the value of computed tomography (CT) in the early diagnosis and follow-up of pyomyositis.

Methods Retrospective clinical and laboratory analysis of one patient.

Results A previously healthy 44-year-old Caucasian workman developed chills and high fever, about $6 \mathrm{~h}$ after an abrasion of the skin over his right hand. Three days later he was admitted to the hospital with hyperthermia $\left(41^{\circ} \mathrm{C}\right)$ and prostration. Physical examination revealed an exquisite tenderness of the right subclavicular area with local warmth, swelling and erythema of the skin, indicating acute inflammation in the chest wall. No evidence of suppuration, cellulitis, lymphangitis, or regional lymphadenopathy was observed in the right upper extremity. Laboratory investigation showed an erythrocyte sedimentation rate of $47 \mathrm{~mm}$, a white blood cell count of $17,380 / \mathrm{mm}^{3}$ with $84 \%$ polymorphs, and a creatine phosphokinase of $1,520 \mathrm{IU} / \mathrm{L}$ (normal range 24-234 IU/L). Multiple blood cultures were negative and chest X-rays were normal. A CT scan of the chest demonstrated marked swelling of the right pectoralis minor muscle. This evidence of acute muscle inflammation supported the presumptive diagnosis of acute pyomyositis, in its invasive stage. Intravenous antibiotics therapy with a combination of clindamycin and ticarcillin/clavulanic acid was started on admission. Fever, tenderness, and other signs of inflammation subsided in a few days with antibiotics alone. No surgical procedure was necessary. After two weeks of hospitalisation the patient was discharged in good condition, but antibiotics were continued oraly for two more weeks. Complete clinical recovery with restoration to normal of all laboratory tests was achieved within one month. A new CT imaging taken four weeks later revealed a complete resolution of the inflammation with no residual deformity of the affected muscle, verifying the favourable outcome of prompt antibiotic treatment.

Conclusion Although the causative microorganism was not identified, there is convincing evidence that this patient had pyomyositis. This case illustrates the value of CT in timely diagnosis and nonsurgical management of pyomyositis, by showing that: a) CT imaging can allow early detection of pyomyositis during its presuppurative phase, b) prompt antibiotic therapy may obviate surgery, and c) besides facilitating an early diagnois, CT is also very useful in the follow-up of pyomyositis. 